\title{
Initial Validation of the Arabic version of the Psychological Flourishing Scale with Egyptian Athletes Students
}

\section{"Dr/ Marei Salama-Younes}

Abstract

Background. In different countries, the psychological flourishing has largely been studied in western cultures (Bakker \& Schaufeli, 2008; Diener et al., 2010; B. L. Fredrickson \& Losada, 2005; Keyes, 2010; Ryff \& Singer, 2003; Silva \& Caetano, 2013). In Arabic culture, in exercise psychology, the Psychological Flourishing Scale (PFS) by means of positive psychology approach has not yet been used. The specific goals of the present research are twofold as follows: i) test the internal reliability and the structure validity of the Arabic version, ii) test its temporal stability and convergent validity. Methods. For that, two studies were conducted. Samples were Egyptian Athletes students $(n=3 r r ; n=1 \wedge l)$. In the first study and in order to verify its structure validity, the EFA and CFA have been performed. In the second study, the temporal stability and convergent validity has been tested. For that, The Arabic versions of the Psychological Flourishing Scale (PFS), Mental Health ContinuumShort Form (MHC-SF), and Satisfaction with Life Scale (SWLS) were administered to the participants. Results. For Egyptian Athletes students, the PFS showed a good internal reliability, structure and convergent validity. However, it has a moderate temporal stability. Conclusions. The Arabic version of the PFS represented good psychometric properties for young athletes. The Arabic version of the Psychological Flourishing Scale could be used in exercise psychology for Egyptian Athletes.

Keywords: CFA, PFS, MHC-SF, Life Satisfaction, College students. Initial Validation of the Arabic version of the Psychological Flourishing Scale with Egyptian Athletes Students

Associate Professor, Department of Psychology, Sociology and Evaluation in Sport Field, Helwan University, Cairo, Egypt \& Associate member, CREAD-ESPE and LAUREPS-CRPCC, Psychology Department, Rennes2 University, Rennes, France. 


\section{Introduction}

Flourishing generally refers to the good life-feeling good and functioning effectively (Huppert \& So, 2013). Although there are no unique or universal definitions of well-being and other positive psychological functioning (Coleman, 2009), numerous models of flourishing have been proposed. For example, Seligman (2011) introduced the PERMA model. Huppert and So (2013) suggested 10 components of flourishing that are the opposite of depression and anxiety. (Diener et al., 2010) developed a brief 8 -item measure of flourishing, designed to assess psychosocial success across several areas of life and to complement measures that focus on affect and life satisfaction.

Based on the assumption that well-being would prevail when pathology was absent; epidemiology has traditionally focused on disease (Huppert \& So, 2013). But a growing body of research challenges this assumption, asserting that mental illness and mental health are two separate, albeit related, continua (Keyes, 2002,
2005). The epidemiology of high levels of wellbeing, referred to here as flourishing, is an important research topic fuelling substantial international interest in its psychometric measurement (L. C. Hone, Jarden, Schofield, \& Duncan, 2014; Keyes, 2007). Flourishing is a positive psychology concept which is a measure of overall life wellbeing and is viewed as important to the idea of happiness (B. L. Fredrickson \& Losada, 2005). Many components and concepts contribute to the overall concept of flourishing and the benefits of a life that can be characterized as flourishing (Huppert \& So, 2013). The emerging field of positive psychology aims to redress this imbalance. In Flourishing, distinguished scholars apply scientific analyses to study the good life, expanding the scope of social and psychological research to include happiness, well-being, courage, citizenship, play, and the satisfactions of healthy work and healthy relationships. Their findings reveal that a sense of meaning and a feeling of richness emerge in life as 
people immerse themselves in activities, relationships, and the pursuit of intrinsically satisfying goals like overcoming adversity or serving one's community through volunteering (Keyes, 2010).

For example, individuals who are flourishing have reported better health, such as the lowest risk of cardiovascular disease and fewest limitations of activities of daily living, and thus unsurprisingly have lower health care utilisation and fewest missed days of work (Keyes, 2007). It is theorised that flourishing and moderate mental health are a source of resilience, acting as a buffer against stressful life events (Keyes, 2002). Catalino and Fredrickson (2011) indicated that relative to individuals who did not flourish or were depressed, individuals who flourished tended to respond with larger spikes in positive emotion following everyday pleasant events. Therefore, flourishing adults have higher levels of motivation to work actively to pursue new goals and are in possession of more past skills and resources. This helps people to satisfy life and societal goals, such as creating opportunities, performing well in the workplace, and producing goods, work and careers that are highly valued in society. This success results in higher satisfaction and reinforces Frederickson's Broaden and Build model, for more positive adults reap more benefits and, are more positive, which creates an upward spiral (Catalino \& Fredrickson, 2011).

Studies have shown that people who are flourishing are more likely to graduate from college, secure "better" jobs, and are more likely to succeed in that job. One reason for this success can be seen in the evidence offered above when discussing languishing: those that flourish have less work absenteeism (Lyubomirsky, King, \& Diener, 2005). Finally, those that are flourishing have more support and assistance from co-workers and supervisors in their workplace. Flourishing has been found to impact more areas than simply the workplace. In particular, community involvement and social relationships have been cited as something that flourishing influences directly. 
For example, those that flourish have been found to volunteer at higher levels across cultures. Moreover, in terms of social support and relationships, studies have shown that there is an association between flourishing and actual number of friends, overall social support, and perceived companionship

(Bakker \& Schaufeli, 2008; Lyubomirsky et al., 2005).

Flourishing from Keyes's concept

Flourishing is a concept that helps the social scientists

and psychologists study and measure fulfilment, purpose, meaning, and happiness. According to Keyes and Annas (2009), for estimating the flourishing, he argues that mental health does not imply an absence of mental illness. Rather, mental health is a separate dimension of positive feelings and functioning._Therefore, Keyes has operationalized many of the symptoms of positive feelings and positive functioning in life by reviewing dimensions and scales of subjective wellbeing and, therefore, creating a definition of flourishing
(Keyes, 2002). He then claims that flourishing is the product of mentally healthy adults having high levels of emotional well-being. They are happy and satisfied. They tend to see their lives as having a life purpose. They feel some degree of mastery environment and accept all parts of themselves. They have a sense of personal growth in the sense that they are always growing, evolving, and changing. Finally, they have a sense of autonomy and an internal locus of control. They chose their fate in life instead of being victims of fate.

Flourishing

has significant positive aspects magnified when compared to languishing adults and when languishing adults are compared to depressed adults. For example, languishing adults have the same amount of chronic disease as those that are depressed whereas flourishing adults are in exceptionally better physical health. Languishing adults miss as many days at work as depressed adults and, in fact, visit doctors and therapists mor e than depressed adults (Keyes, 2005). 
For evaluating the flourishing aspect, the Mental Health Continuum-Long Form (MHC-LF) consisted of 40 items evaluating 14 facet of well-being. The Short form (MHC-SF) consists of only 14 items were chosen as the most prototypical items representing the 14 facet. One item from each has been chosen. Three items were chosen (happy, interested in life, and satisfied) to represent emotional well-being; five items were chosen to represent the social well-being (social integration, social acceptance, social contribution, social coherence and social actualization); six items were chosen to represent the psychological well-being (selfacceptance, positive Autonomy, environmental mastery, life purpose and personal growth). MHC-SF has been used in many cultures (Keyes et al., 2008; Lamers, Westerhof, Bohlmeijer, ten Klooster, \& Keyes, 2011; Petrillo, Capone, Caso, \& Keyes, 2014; M SalamaYounes \& Ismaïl, 2011).

\section{Flourishing from Diener's concept}

Diener et al. (2010) have recently published new, easily useable questionnaires to assess psychological flourishing, positive and negative emotions. These questionnaires could be very helpful. A high level of mental health provides at least three overlapping benefits - it feels pleasanter and more enjoyable, it promotes better relationships and functioning, and it provides greater resilience (better-shock absorbers) to guard against slipping back into mental distress. These advantages have been demonstrated both for Keyes's multilevel definition of flourishing and for Fredrickson's positive to negative emotions ratio (B. Fredrickson, 2009; B. L. Fredrickson \& Losada, 2005).

Moreover, (Diener et al., 2009) developed the Psychological Flourishing Scale (PFS). It is strongly associated with other psychological well-being scales and they argue fairly convincingly that their scale of positive \& negative experience (SPANE) improves on previous measures of positive $\&$ negative emotion. The Psychological Flourishing Scale (PFS), based on recent theories of psychological and 
social well-being. The PFS is designed to measure socialpsychological prosperity, to complement existing measures of subjective well-being (Diener et al., 2009). A number of psychological theories of human flourishing have been developed, and the authors devised a brief measure to capture major aspects of this type of "prosperity." Based on earlier humanistic psychology theories, (Ryan \& Deci, 2000; Ryff \& Singer, 1998) suggest that there are several universal human psychological needs, such as the need for competence, relatedness, and self-acceptance, and several of these characteristics are assessed by the Psychological Flourishing Scale. The measure has good psychometric properties, and is strongly associated with other psychological well-being scales (Diener et al., 2009). It has been translated and used in different cultures (L. Hone, Jarden, \& Schofield, 2014; Silva \& Caetano, 2013; Sumi, 2014; Tang, Duan, Wang, \& Liu, 2014; Villieux, Sovet, Jung, \& Guilbert, 2016).

\section{Objective of studies}

The purpose of the present research was to test the psychometric properties of the Arabic version of the Psychological Flourishing Scale (PFS, Diener et al., 2010). Two studies have been conducted by three different samples. The objective of the two studies presented involving more than 550 participants, is firstly to test the internal reliability and structure validity of the Arabic version in psychological flourishing scale. The objective of the second study was to test the temporal stability and convergent validity among university students. For that, the relationships between psychological flourishing, Emotional, psychosocial flourishing, MHC-SF total score, and life satisfaction have to be explored. Transcultural validation methodology procedure developed by Vallerand (1989) has been met. In more detail, the first study was designed to test the structural validity of the scale using both of the exploratory factor analysis (EFA) and the confirmatory factor analysis (CFA). Here, the internal consistency was, twice, verified. The purpose of the second study was to test the temporal stability; the 
convergent validity by identifying the relationship between psychological flourishing scale proposed by Diener et al., 2009, the different characteristics of Keyes flourishing scale (Pavot \& Diener, 2008) and life satisfaction Pavot and Diener (2008). For that, the Flourishing scale has been translated into Arabic.

\section{Translation of psychological flourishing scale}

The original scale is comprised of 8 items evaluating only one dimension. The scale provides a single psychological flourishing score that can be used to provide useful feedback for how to improve one's life and provide useful stimulus for selfreflection. The response mode is a seven point Likert scale ranging from (1) "totally not agree" to (7) "totally agree". Two bilingual conducted a reverse translation procedure adopted to achieve the preliminary version of psychological flourishing scale in Arabic. One of them translated the scale from English to Arabic, and the other from Arabic to English. A third teacher, expert in the field, assessed the concordance of the two versions. To ensure the clarity of each item, 17 students of the third academic year, were then sought. They completed the questionnaire and an interview with each of them helped validate the translation. The results showed that the 8 item was clear. This first experimental version was thus composed of 8 items, as the original one.

Study 1: Structural Validity of the Experimental Arabic Version of the Flourishing scale Methods Participants and Procedure

Paper version of the Psychological Flourishing scale was administered during a university session. For testing the structure validity using the EFA and the CFA, two physical education students from Menoufia University samples were replied to this version. They were $37 r$ students were practicing different sport activities (87\% collective $v s .13 \%$ individual). The first sample was physical education students from Menoufia University. The first sample was167 physical education students: $5 \mathrm{~V}$ females $(32.57 \%)$ and $11^{\wedge}$ males (67.43\%), aged between 17 and 26 years $(\mathrm{M}=19.94, S D=$ 
2.27). The second sample was 207 physical education students who participated voluntarily: 62 females (29.95 $\%)$ and 145 males (70.05\%), between the ages of 17 and 22 years $(\mathrm{M}=19.94, S D=2.27)$.

\section{Measure}

\section{Psychological}

Flourishing Scale (PFS). It is a brief 8-item summary measure of the respondent's self-perceived success in important areas such as relationships, self-esteem, purpose, and optimism. The scale provides a single psychological well-being score and can be used to provide useful feedback for how to improve one's life and provides useful stimulus for selfreflection. The PFS is best used with individuals without clinic disorders, but rather with individuals seeking to enhance an already relatively adaptive lifestyle.

For the EFA, the data collection was in the period of 3-28 April, 2013; and for the CFA, it was in the period from 2-17 October, 2013. The two samples have been informed about the objective of the study, their participation was voluntary and they could withdraw at any time. Both oral and written instructions were given regarding items understanding (i.e., there was no right or wrong answer to the questions and they should freely state what they think), and they were reassured about the confidentiality of their responses.

\section{Statistical analysis}

Missing values (representing 1\% of the total data file) were replaced using a regression imputation procedure. The SPSS 21 was used to perform the correlation matrix, internal consistency and EFA. The LISREL 8.8 software Pavot and Diener (2008) was used to test the CFA.

\section{Results and Discussion}

\section{Analysis \\ Exploratory Factor}

The data were screened for non-normality, and no problematic trend was detected. To ensure univariate normality, Kline (1998) suggested cut-off of absolute values of 3.0 and 8.0 for skewness and kurtosis, respectively. Univariate skewness ranged from -1.27 to .73 , and univariate kurtosis ranged from -.98 to 1.19 , indicating that the responses were relatively normally 
distributed. The internal reliability of the psychological flourishing scale was assessed by using Cronbach's coefficients. It was .82.

In order to assess the structure validity of the PFS using the (EFA), Principal Component Analysis was performed. This technique comparable to factor analysis aims to examine the underlying factors of a set of items. According to Tabachnick and Fidell (2007), items that did not reach a minimum weight of .40 on one factor were removed. At this first analytic step, a single factor was expected to explain the variation in the scale's variables for the 8 items. Items did reach a weight ranging between .61 to .84. The component factor account revealed to $60.39 \%$ of the variance. The initial eigenvalue was 4.83 . The results revealed an accurate KMO index of .89 . It indicates satisfactory correlations between items. Furthermore, the results of the Bartlett's test of sphericity are significant ( $p>.000)$. Therefore, not all the correlations are equal to zero. We decided then to proceed with the analyses (Table 1).

\section{Table (1)}

\section{Descriptive analysis, and unidimensionality of the Flourishing} scale

\begin{tabular}{c|c|c|c|c|c|c|c|c}
\hline \hline Items & $\begin{array}{c}\text { Factor } \\
\text { loading }\end{array}$ & Mean & $\begin{array}{c}\text { St. } \\
\text { Dev. }\end{array}$ & $\begin{array}{c}\text { Correlated } \\
\text { item total } \\
\text { correlation }\end{array}$ & $\begin{array}{c}\text { Squared } \\
\text { multiple } \\
\text { correlation }\end{array}$ & Skew & Kurt & $\begin{array}{c}\text { Cronbach } \\
\text { Alpha }\end{array}$ \\
\hline \hline F1 & .61 & 4.88 & 1.99 & .52 & .34 & -.73 & -.58 & .91 \\
\hline F2 & .79 & 5.62 & 1.88 & .72 & .59 & -1.39 & .72 & .89 \\
\hline F3 & .74 & 5.22 & 1.67 & .67 & .48 & -.87 & -.02 & .89 \\
\hline F4 & .83 & 5.35 & 1.80 & .76 & .61 & -.97 & -.07 & .89 \\
\hline F5 & .84 & 5.27 & 1.85 & .77 & .62 & -1.01 & .02 & .88 \\
\hline F6 & .84 & 5.95 & 1.97 & .77 & .66 & -.67 & -.79 & .88 \\
\hline F7 & .79 & 5.55 & 1.84 & .71 & .55 & -1.39 & .89 & .89 \\
\hline F8 & .75 & 5.06 & 1.95 & .66 & .58 & -.84 & -.40 & .91 \\
\hline total & 4.83 & 5.24 & 1.86 & & & & & .91 \\
\hline \% of & 60.39 & & & & & & & \\
variance & & & & & & & & \\
\hline \hline
\end{tabular}

Note $n=167$ 


\section{Confirmatory Factor Analysis}

The objective of this study was to test the structural validity of the PFS scale. The unidimensional model has been tested using CFA performed with LISREL 8.8 software Jöreskog and Sörbom, (1996). In the second step, the internal consistency of factor has also been evaluated.

Data were firstly screened for nonnormality, and no problematic trend was detected. Univariate skewness ranged from -1.02 to 1.01 , and univariate kurtosis ranged from -.98 to 1.19 , indicating that the responses were relatively normally distributed. In addition, relative multivariate kurtosis as reported by the output from LISREL 8.8 (Joreskog and Sorbom 1996) equalled 1.14. Whereas, there is no standard cut-off for this index, $\mathrm{Hu}$ and Bentler (1998) recommended that multivariate normality can be assumed if this value is less than 3 . So, the distribution of variables being normal a matrix of productmoment correlations was generated to evaluate the models using the maximum robust-likelihood method (MLrobust).
Moreover, in the present study, we tested the internal consistency by SPSS software. It was assessed by calculating the Cronbach's coefficients. The values of 0.70 or greater were considered satisfactory. We performed then the CFA to assess the passion scale. The intention was to indicate if the model fits well the data. There are varying suggestions in the literature about the number, type, and cut-off values for goodness-of-fit required to be reported for CFA (Keyes et al., 2008). A popular recommendation is to present three of four indices from different areas. Accordingly, we report several goodness-of-fit indicators including GFI (Goodness-ofFit Index), NFI (Normed Fit Index), RMR (Root Mean Square Residual), RMSEA (Root Mean Square Error of Approximation), and $\chi^{2} / \mathrm{df}$. The recommended cut-off values for acceptable values are $\geq 0.90$ for GFI, AGFI and NFI. The RMR and RMSEA test the fit of the model to the covariance matrix. As a guideline, values below 0.05 indicate a close fit and values below 0.11 are an acceptable 
fit. The value of 2 alone may be used as an index, but 2 divided by the degrees of freedom (2/df) reduces its sensitivity to sample size (cutoff values $<2$ to 5 ).

Table (2)

Descriptive statistics and indicator inter-correlations for different variables

\begin{tabular}{l|c|c|c|c|c|c|c}
\hline \hline \multicolumn{1}{c|}{ Variables } & $\mathbf{1}$ & $\mathbf{2}$ & $\mathbf{3}$ & $\mathbf{4}$ & $\mathbf{5}$ & $\mathbf{M}$ & SD \\
\hline \hline FS & - & & & & & 5.09 & 1.11 \\
Emotion-MHC-SF & $.22^{*}$ & - & & & & 4.57 & 1.24 \\
Function-MHC-SF & $.29 *$ & $.53^{*}$ & - & & & 4.39 & 0.92 \\
MHC-SF-Total & $.32^{*}$ & $.77^{*}$ & $.94^{*}$ & - & & 4.32 & 0.94 \\
SWLS & $.55^{*}$ & $.35^{*}$ & $.32^{*}$ & $.33^{*}$ & - & 4.52 & 1.02 \\
\hline \hline
\end{tabular}

Note $n=207^{*} p<.01$

In our study, the main goal of the CFA was to test the unidimensionality of the Arabic version of PFS; it means that the 8 items saturate only one factor. Results presented in table 2 , show that this model fits with the data. Results of CFA revealed a satisfactory fit to the data in terms of $\chi 2 / \mathrm{df}$ ratio, GFI, CFI, NFI, RMR, and RMSEA. The $\chi^{2}(767.03,28, \mathrm{~N}=207)=$ 27.93, $p=0.03$, Normed Fit Index $\quad(\mathrm{NFI})=0.95$, Comparative Fit Index $(\mathrm{CFI})=$ 0.94, Root-Mean-Square Error of Approximation (RMSEA)= .04 [.03; .05], Goodness-of-Fit Index (GFI) = 0.97, Comparative Fit Index $(\mathrm{CFI})=$ 0.96, Adjusted Goodness-of-Fit Index $(\mathrm{GFI})=0.96$, Root Mean Square Residual (RMR) =

0.04. The Goodness of Fit was acceptable in terms of $\chi 2 / \mathrm{df}$ ratio, GFI, NFI, RMR, and RMSEA. Finally, the reliability of the scale was assessed using Cronbach's Alpha in accordance with standard procedures and for the whole of items. It was acceptable 0 . 89. We concluded that the Arabic version of PFS had an acceptable factor structure and internal consistency as well as the original version.

Study 2: Temporal Stability and Convergent Validity of the Psychological Flourishing Scale

The structural validity and reliability are not sufficient to fully validate a psychometric scale. It is also important to test its relationships with other psychological constructs with 
which it is presumed to be correlated. The objective of this study is twofold. Firstly, we aim to verify the temporal stability of the PFS across the time. In accordance with the theoretical framework, we have not found indication for the temporal stability for the PFS.

The second study aims to test the relationships between psychological flourishing, emotional, psychosocial flourishing, MHC-SF total score and life satisfaction will be investigated. For that, scores on flourishing scale are presumed to be correlated with satisfaction with life scale (Diener et al., 2010) . In addition, we assumed that PFS would also be correlated with hedonic and eudaimonic wellbeing.

\section{Participants and Procedure}

The paper version was administered twice at a university to unpaid and voluntary students who signed a consent form. They were $1 \wedge$ I at time 1 , and the re-test performed 3 weeks apart had to match $1 \mathrm{Vr}$ students between time 1 and time 2. They were from physical education faculty from Helwan University. They aged from 17 to $26(\mathrm{M}=23.03, S D=2.92)$.
The remaining students either did not wish to participate or did not present in the second phase of the study. In addition to this questionnaire, at time 1 , students completed two scales: the Satisfaction With Life Scale (Diener, Emmons, Larsen, \& Griffin, 1985) and MHC-SF (Keyes, 2007).

Psychological Flourishing Scale (FS) The PFS is a brief 8 -item which we are testing its reliability and convergent validity in the present research. Internal consistency reliability (Cronbach's $\alpha$ coefficient) for the total score in this sample was 0.89 .

\section{Mental Health Continuum- Short Form, MHC-SF} (Keyes, 2006). The Short Flourishing scale is a 14-item measure that assesses several individual and social aspects of well-being, including positive relationships with others, environmental mastery, selfacceptance, purpose in life, personal growth, autonomy, acceptance of others, a sense of belonging to a community, a belief that social groups can evolve positively, a sense that one's life is useful to society, and interest in social life. Internal consistency reliability (Cronbach's $\alpha$ coefficient) for 
the total score in this sample was 0.77 .

The Mental Health Continuum - Short Form (MHC-SF) is a 14 item selfreport questionnaire that assesses hedonic and eudaimonic well-being (Keyes, 2002). Participants rate the frequency of each feeling in the past month on a 6-point Likert scale (from never to every day). It was shown that three items form the Emotional Wellbeing subscale, which assesses positive emotions towards one's life "Satisfied with life", five items form the Social Wellbeing subscale ("That you had something important to contribute to society') and six items form the Psychological Wellbeing subscale ("That you liked most parts of your personality"), which assesses engagement and functioning in one's social and private life. Cronbach's alpha in the current sample was satisfactory (.76).

Satisfaction with Life Scale (SWLS). The SWLS is a global measure of life satisfaction. The SWLS composes of five items. A 7 point Likert scale was used ranging from "strongly disagree" (1-point) to "strongly agree" (7-point). The SWLS is a measure of life satisfaction developed by (Pavot \& Diener,
2008)The internal consistency was 0.84 .

\section{Results and Discussion}

Taken together, the results showed good correlations for the test-retest reliability $(.62 \leq \mathrm{r} \geq .82, p<$ .001).). Using the data set of the second study, we concluded that temporal stability for flourishing scale is acceptable. According to Cohen (1988), these correlations ensure moderate to high relation (Cohen, 1988). They are consistent with the expected stability of the scale as supposed to be closer more state than trait. Cronbach's alpha of the scale at time 1 is .82 while at time 2 , it is .88 . Secondly, there is significant and positive relationship between total score of PFS, short flourishing (emotional and functional) scores, and life satisfaction. Thus, without knowing the direction; an individual who feels high life satisfaction in their life will have high scores on emotional and functional flourishing. In other word, if he or she feels emotionally and/or functionally flourished in their life, he or she feels life satisfied. 
Table (3)

Descriptive statistics, Cronbach alpha and indicator intercorrelations for items scale

\begin{tabular}{l|c|c|c|c|c|c|c|c|c|c|c|c|c}
\hline \hline Items & 1 & 2 & 3 & 4 & 5 & 6 & 7 & 8 & $\mathrm{M}$ & SD & Skew & Kurt & $\begin{array}{c}\text { Cronbach } \\
\text { Alpha }\end{array}$ \\
\hline \hline $\mathrm{F} 1$ & - & & & & & & & & 5.08 & 1.51 & -.94 & .41 & .86 \\
\hline $\mathrm{F} 2$ & $.34^{*}$ & - & & & & & & & 5.79 & 1.46 & -1.27 & 1.19 & .85 \\
\hline $\mathrm{F} 3$ & $.29^{*}$ & $.48^{*}$ & - & & & & & & 5.47 & 1.48 & -1.26 & 1.16 & .86 \\
\hline $\mathrm{F} 4$ & $.40^{*}$ & $.33^{*}$ & $.33^{*}$ & - & & & & & 5.41 & 1.54 & -.85 & .18 & .85 \\
\hline $\mathrm{F} 5$ & $.43^{*}$ & $.50^{*}$ & $.39^{*}$ & $.62^{*}$ & - & & & & 5.37 & 1.59 & -1.13 & .42 & .84 \\
\hline $\mathrm{F} 6$ & $.37^{*}$ & $.42^{*}$ & $.34^{*}$ & $.42^{*}$ & $.61^{*}$ & - & & & 5.12 & 1.63 & .73 & -.34 & .84 \\
\hline $\mathrm{F} 7$ & $.37^{*}$ & $.54^{*}$ & $.49^{*}$ & $.46^{*}$ & $.49^{*}$ & $.58^{*}$ & - & & 5.58 & 1.53 & -1.22 & -.98 & .84 \\
\hline $\mathrm{F} 8$ & $.45^{*}$ & $.37^{*}$ & $.38^{*}$ & $.49^{*}$ & $.47^{*}$ & $.56^{*}$ & $.61^{*}$ & - & 5.10 & 1.46 & -.72 & .33 & .84 \\
\hline Total & & & & & & & & & & & & & .86 \\
\hline \hline
\end{tabular}

Note $n=181^{* *} p<.01 * p<.05$ According to Cohen (1988) recommendations, correlation under .10 is "low", between .10 and .30 is "moderate", and under .50 is "high". Table 2 shows the moderate correlations were possible to observe. To resume, these results ensure adequate convergent validity of the PFS. We conducted a student's Ttest to check whether there was a significant difference between time 1 and 2 scores on the total score. Results show a significant difference for PFS $(\mathrm{t}=2.22 ; p>.05)$. These results can be justified by the fact that the retest was carried out during the last class before exams of the second semester. The difference between time 1 and time 2 is relatively high. The explanation may come from the presence of an adjustment disorder with respect to stress, which is very high amongst the students at this time of the year.

\section{Discussion}

Starting from the first aim in this study, in the original psychological flourishing scale (PFS) composed of 8 items. Although there is an exploratory study for the Arabic version, the first study confirms the structure validity and reliability of the flourishing scale as a unidimensional construct. Recently, the same structure has been confirmed in many cultures (e.g., Chinese, France, Japanese, News land, Portugal). Results support the unidimensional structure for the psychological flourishing 
scale in Arabic context. Furthermore, correlations between flourishing scale and the emotional well-being, functional well-being and life satisfaction supported the convergent validity of the Arabic Scale. The Psychological Flourishing Scale emerged as unidimensional scale that shares measurement factors with more established hedonic and eudemonic well-being scales.

The first objective of the present research aims to test if the PFS could be unidimensional. Collected data have confirmed this hypothesis. However, it is impossible to determine the directionality of causality with respect to the proposed model (flourishing, emotional, functional well-being and life satisfaction). Findings of the second study show that high flourishing score led to equally significant and high levels of emotional, functional wellbeing and life satisfaction. However, low flourishing score led to significant and low level of emotional, functional wellbeing and life satisfaction. Using the same data set of the second study, the more people have a high flourishing level, the more they are life satisfied, feeling more happy and psychosocial well-being in their life.

Moreover, practicing the favourite physical activity brings some people intrinsic pleasure and joy. These people feel good about themselves during and after practicing, find their favourite activity to be in harmony with their life style. But physical education students, who practicing a large variety of sports activities in their sessions, feel an uncontrollable urge to engage in their favourite practicing and feel more conflict between their passion and other sport activities (Keyes, Shmotkin, \& Ryff, 2002)

It can be stated that the Psychological Flourishing Scale has not been validated for sample with a wide demographic range. Although that the three samples leads to the assumption that the sample is unlikely to be representative for the whole population being studied. The samples of studies represent less than 5\% of Egyptian Athletes students. In addition, samples issue from only two physical education faculty. Nevertheless, the 
validation of flourishing scale with a variety of age, genre and demographical level samples would be of high value.

These findings suggest that flourishing, emotional, functional well-being and life satisfaction significantly and positive correlated. However, the second study had two important limitations: it relied on participants' recollection of their social, economic situation and life satisfaction. Thus, many raisons could probably lead to these results. _ It is possible that the economic resources, urban rivals, cultural assets or marital situation biases explain these findings. In the present study, collected data was done just after they were finishing their academic session. It would probably have an effect in their concentration level. A longitudinal and causality study would be more beneficial for the college students.

\section{Conclusion}

Study of flourishing of life in the field of sports is relatively new (Mareï SalamaYounes, 2011a, 2011b; Mareï Salama-Younes, Ismaill, Montazeri, \& Roncin, 2011). It contains concepts about psychological well-being, from the perspectives of positive psychology. Unlike most of the psychological studies about sports, which usually focus on achieving sporting success and excellence, the perspective of our research emphasises about the manner of athletes on their strengths to become flourished whole persons.

Objectives of these studies were (i) to test the factor structure of the psychological flourishing scale (PFS) using the exploratory and confirmatory factor analysis; (ii) to test temporal stability for flourishing scale and convergent validity by the relationships with emotional, functional well-being and life satisfaction. For that, three different samples practicing regularly physical activities have been solicited in two studies. Findings from the first study show a good structure of flourishing scale. The objective of the second study was to test the convergent validity by using the correlation between emotional, functional wellbeing and life satisfaction. Data confirmed the hypothesis. The results of this study should be interpreted in the light of a number of limitations. Respondents were not asked 
about the duration, frequency, or intensity for their physical activity practice. Some limitations should then be kept in mind when interpreting the current findings. First, it is impossible to determine the directionality of causality with respect to the proposed model. Consequently, researchers should try to replicate the present findings using experimental designs in order to clearly establish the directionality of effects. This experimental design should be in sequence of times. In the present study, we passed questionnaire of three scales just in beginning or after finishing the physical activity session. Second, the sample contained a disproportion of men and women from two universities. This drawback did not permit us to investigate the issue of gender differences and it was not planning as objective. Future research should look into this issue given that gender differences have been reported in the flourishing not only as a cause but as mediator variable. Flourishing may also affect one's physical and/or mental health and academic performance. A path analysis for these variables would be interesting.

Final conclusion shows the quite extensive examination and validation of the Psychological Flourishing Scale suggests that it is a new, short and useful tool to assess the psychological well-being. Psychological flourishing can be regarded as a complementary element to predict a full state of general health. It comprises the fulfilment of human needs and the appreciation of positive life aspects. The elements of the scale may serve as an inducement to strive for an adequate compliance of personal goals which correspond to one's individual values. After all, subjective well-being is not only defined by the presence of physical health or the absence of mental illness, but also to a not inconsiderable extent by the way how positively reinforcing behaviours are implemented. For the Psychological Flourishing Scale, the conclusions that came up with the empirical examinations argue for the presence of a valid and reliable scale in Egyptian culture. In future studies, it is important to 
examine the prediction of PS on cognitive, emotional and behaviour consequences for Egyptian Athletes. It is available in the following website It is in the flowing website

http://internal.psychology.illino is.edu/ ediener/FS.html

\section{References}

1- Bakker, A. B., \& Schaufeli, W. B. (2008). Positive organizational behavior: Engaged employees in flourishing organizations. Journal of Organizational Behavior, 29(2), 147-154.

2- Catalino, L. I., \& Fredrickson, B. L. (2011). A Tuesday in the life of a flourisher: the role of positive emotional reactivity in optimal mental health. Emotion, 11(4), 938.

3- Cohen, J. (1988). Statistical power analysis: A computer program: Routledge.

4- Coleman, J. (2009). Well- being in schools: empirical measure, or politician's dream? Oxford Review of Education, 35(3), 281-292.

5- Diener, E., Emmons, R. A., Larsen, R. J., \& Griffin, S. (1985). The satisfaction with life scale. Journal of personality assessment, 49(1), 71-75.

6- Diener, E., Wirtz, D., Biswas-Diener, R., Tov, W., Kim-Prieto, C., Choi, D.-w., \& Oishi, S. (2009). New measures of well-being: Springer.

7- Diener, E., Wirtz, D., Tov, W., Kim-Prieto, C., Choi, D.w., Oishi, S., \& Biswas-Diener, R. (2010). New well-being measures: Short scales to assess flourishing and positive and negative feelings. Social Indicators Research, 97(2), 143-156.

8- Fredrickson, B. (2009). Positivity: Three Rivers Press (CA).

9- Fredrickson, B. L., \& Losada, M. F. (2005). Positive affect and the complex dynamics of human flourishing. American psychologist, 60 (7), 678.

10- Hone, L., Jarden, A., \& Schofield, G. (2014). Psychometric properties of the Flourishing Scale in a New Zealand sample. Social Indicators Research, 119(2), 1031-1045.

11- Hone, L. C., Jarden, A., Schofield, G. M., \& Duncan, S. (2014). Measuring flourishing: The impact of operational definitions on the prevalence of 
high levels of wellbeing. International Journal of Wellbeing, 4 (1).

12- Hu, L.-t., \& Bentler, P. M. (1998). Fit indices in covariance structure modeling: Sensitivity to underpara meterized model misspecification.Psychological Methods, 3(4), 424.

13- Huppert, F. A., \& So, T. T. (2013). Flourishing across Europe: Application of a new conceptual framework for defining well-being. Social Indicators Research, 110(3), 837-861.

14- Keyes, C. L. (2002). The mental health continuum: From languishing to flourishing in life. Journal of health and social behavior, 207-222.

15- Keyes, C. L. (2005). Mental illness and/or mental health? Investigating axioms of the complete state model of health. Journal of consulting and clinical psychology, 73(3), 539.

16- Keyes, C. L. (2007). Promoting and protecting mental health as flourishing: a complementary strategy for improving national mental health. American Psychologist, 62(2), 95.
17- Keyes, C. L. (2010). Flourishing: Wiley Online Library.

18- Keyes, C. L., \& Annas, J. (2009). Feeling good and functioning well: Distinctive concepts in ancient philosophy and contemporary science. The Journal of Positive Psychology, 4(3), 197-201.

19- Keyes, C. L., Shmotkin, D., \& Ryff, C. D. (2002). Optimizing well-being: the empirical encounter of two traditions. Journal of personality and social psychology, 82(6), 1007.

20- Keyes, C. L., Wissing, M., Potgieter, J. P., Temane, M., Kruger, A., \& van Rooy, S. (2008). Evaluation of the mental health continuum-short form (MHC-SF) in setswana- speaking South Africans. Clinical Psychology \& Psychotherapy, 15(3), 181192.

21- Kline, R. B. (1998). Software review: Software programs for structural equation modeling: Amos, EQS, and LISREL. Journal of psychoeducational assessment, 16(4), 343-364.

22- Lamers, S., Westerhof, G. J., Bohlmeijer, E. T., ten Klooster, P. M., \& Keyes, C. L. (2011). Evaluating the 
psychometric properties of the mental health continuumshort form (MHC-SF). Journal of Clinical Psychology, 67(1), 99-110.

23- Lyubomirsky, S., King, L., $\&$ Diener, E. (2005). The benefits of frequent positive affect: does happiness lead to success? Psychological bulletin, 131(6), 803.

24- Pavot, W., \& Diener, E. (2008). The satisfaction with life scale and the emerging construct of life satisfaction. The Journal of Positive Psychology, 3(2), 137-152.

25- Petrillo, G., Capone, V., Caso, D., \& Keyes, C. L. (2014). The Mental Health Continuum-Short Form (MHC-SF) as a measure of well-being in the Italian context. Social Indicators Research, 121(1), 291-312.

26- Ryan, R. M., \& Deci, E. L. (2000). The darker and brighter sides of human existence: Basic psychological needs as a unifying concept. Psychological inquiry, 11(4), 319-338.

27- Ryff, C. D., \& Singer, B. (1998). The contours of positive human health. Psychological inquiry, 9(1), 128.
28- Ryff, C. D., \& Singer, B. (2003). Flourishing under fire: Resilience as a prototype of challenged thriving.

29- Salama-Younes, M. (2011a). Positive mental health, subjective vitality and satisfaction with life for French physical education students. World Journal of Sport Sciences, 4(2), 90-97.

30- Salama-Younes, M. (2011b). Towards a positive sport psychology: A prospective investigation in physical practice. World Journal of Sport Sciences, 4(2), 104-115.

31- Salama-Younes, M., \& Ismaïl, A. (2011). Validation of factor structure of mental health continuum (MHC-SF) for physically active older adults. World Journal of Sport Sciences, 4(1), 20-30.

32- Salama-Younes, M., Ismail, A., Montazeri, A., \& Roncin, C. (2011). Factor structure of the French Ryff's Psychological Well-Being Scales for active older adults The human pursuit of wellbeing (pp. 203-212): Springer. Silva, A. J., \& Caetano, A. (2013). Validation of the flourishing scale and scale of positive and negative experience in Portugal. Social 
Indicators Research, 110(2), 469-478.

33- Sumi, K. (2014).

Reliability and validity of Japanese versions of the flourishing scale and the scale of positive and negative experience. Social Indicators Research, 118(2), 601-615.

34- Tabachnick, B., \& Fidell, L. (2007). Multivariate analysis of variance and covariance. Using multivariate statistics, 3, 402-407.

35- Tang, X., Duan, W., Wang, Z., \& Liu, T. (2014). Psychometric evaluation of the simplified Chinese version of flourishing scale. Research on Social Work Practice, 1049731514557832.
36- Vallerand, R. (1989). Toward a methodology for the transcultural validation of psychological questionnairesImplications for Studies in the french language. Canadian Psychology-Psychologie

Canadienne, 30(4), 662-680. 37- Villieux, A., Sovet, L., Jung, S.-C., \& Guilbert, L. (2016). Psychological flourishing: Validation of the French version of the Flourishing Scale and exploration of its relationships with personality traits. Personality and Individual Differences, 88, 1-5. 